\title{
Vibrational overtone excitation in electron mediated energy transfer at metal surfaces $\dagger$
}

\author{
Russell Cooper, ${ }^{a}$ Igor Rahinov, $\dagger^{a}{ }^{a}$ Zhisheng $\mathrm{Li}^{a}{ }^{a}$ Daniel Matsiev, ${ }^{a}$ Daniel J. Auerbach ${ }^{b}$ and Alec M. Wodtke ${ }^{* a}$ \\ Received 20th January 2010, Accepted 8th April 2010 \\ First published as an Advance Article on the web 24th May 2010 \\ DOI: 10.1039/c0sc00141d
}

\begin{abstract}
Vibrational overtone excitation is, in general, inefficiently stimulated by photons, but can under some circumstances be efficiently stimulated by electrons. Here, we demonstrate electron mediated vibrational overtone excitation in molecular collisions with a metal surface. Specifically, we report absolute vibrational excitation probabilities to $\nu=1$ and 2 for collisions of $\mathrm{NO}(\nu=0)$ with a $\mathrm{Au}(111)$ surface as a function of surface temperature from 300 to $985 \mathrm{~K}$. In all cases, the observed populations of vibrationally excited $\mathrm{NO}$ are near those expected for complete thermalization with the surface, despite the fact that the scattering occurs through a direct "single bounce" mechanism of sub-ps duration. We present a state-to-state kinetic model, which accurately describes the case of near complete thermalization (a regime we call the strong coupling case) and use this model to extract state-to-state rate constants. This analysis unambiguously shows that direct vibrational overtone excitation dominates the production of $\nu=2$ and that, within the context of our model, the intrinsic strength of the overtone transition is of the same order as the single quantum transition, suggesting a possible way to circumvent optical selection rules in vibrational pumping of molecules. This result also suggests that previous measurements of vibrational relaxation of highly vibrationally excited NO exhibiting highly efficient multi-quantum jumps $(\Delta \nu \sim-8)$ are mechanistically similar to vibrational excitation of $\mathrm{NO}(\nu=0)$.
\end{abstract}

\section{Introduction}

The physics and chemistry of interfaces plays an important role in the conversion of energy between its various forms including electrical, photonic, chemical, and thermal. ${ }^{1}$ For example in solar cells, the likelihood of photon-initiated charge-separation is strongly influenced by interfacial phenomena. ${ }^{2,3}$ While still poorly understood, there is little doubt that a structure-function relationship is central to surface reactivity ${ }^{4}$ including heterogeneous catalysis. ${ }^{5}$ Furthermore, reports that the exothermicity of chemical reactions at surfaces results in electrical currents inspire new concepts for sensor ${ }^{6}$ and energy conversion ${ }^{7}$ technology.

Developing better understanding of interfacial energy conversion at the atomic level is particularly promising in studies at the gas-solid interface. Here, sophisticated dynamics methods including molecular beams ${ }^{8}$ and laser spectroscopy ${ }^{9}$ can be combined with surface analytical probes to create well defined experiments, the observations of which can be directly compared to first principles theory. ${ }^{10-12}$ This approach has led to significant insights which have been reviewed on several

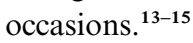

The interconversion of energy between molecular vibration and solid excitation is of particular interest due to the close

${ }^{a}$ Dept. of Chemistry and Biochemistry, University of California Santa Barbara, Santa Barbara, CA, 93106-9510,USA. E-mail: wodtke@chem. ucsb.edu

${ }^{b}$ GRT Inc., 861 Ward Drive, Santa Barbara, CA, 93111, USA

$\dagger$ Electronic supplementary information (ESI) available: The Mathematica Notebook used to analyze the data in this edge article using the multi-quantum kinetic model. See DOI: 10.1039/c0sc00141d

\$ Present address: Department of Particle Physics and Astrophysics, Weizmann Institute of Science, Rehovot 76100, Israel. relationship between molecular vibration and bond dissociation. Furthermore, it is here that some of the clearest evidence has become available that molecular interactions at surfaces are not purely mechanical in nature, ${ }^{16,17}$ but can also interact strongly with electrons in the solid, ${ }^{18}$ even resulting in electron emission when the transferred vibrational energy is greater than the solid's work function. ${ }^{19-21}$ In contrast, when this quantity is less than the solid's band gap, vibrational energy transfer to or from the solid is less efficient. ${ }^{22-24}$

A recent theoretical treatment of electronically non-adiabatic interactions ${ }^{10}$ has been remarkably successful at reproducing experimental observations including rotational cooling with vibrational relaxation ${ }^{25}$ and vibrational insensitivity to trapping. ${ }^{26}$

Perhaps the most interesting agreement between experiment and theory involves multi-quantum $(\Delta \nu \sim-8)$ vibrational relaxation of highly vibrationally excited $\mathrm{NO}(\nu=15)$ in collisions with a $\mathrm{Au}(111)$ surface. Here, sub-ps time-scale electron hopping between $\mathrm{NO}$ and $\mathrm{Au}$ results in efficient high overtone vibrational relaxation and creation of a single correspondingly high energy electron hole pair (EHP). ${ }^{10,18}$ By comparison, photon emission from $\mathrm{NO}(\nu=15)$ is dominated by $\Delta \nu \sim-1$ transitions. ${ }^{27}$ These results demonstrate a crucial difference between electron mediated and photon mediated vibrational transitions; namely, the probability of "high overtone" transitions can be much larger in the former than in the latter. This begs the question to what extent electron mediated processes might be used to circumvent optical selection rules for vibrational overtone excitation, providing an avenue for direct pumping of many quanta into molecular vibration on the sub ps time-scale. 
In principle, the mechanism of multi-quantum vibrational relaxation ${ }^{10}$ just described is consistent with one originally discussed $^{28}$ in connection to single quantum vibrational excitation of small molecules on metal surfaces: ${ }^{16,29}$ however, the direct observation of multi-quantum vibrational excitation has never been reported within the context of an electron mediated energy transfer mechanism. ${ }^{30}$

In this edge article, we present results of new experiments where electron mediated multi-quantum vibrational excitation is seen for the first time. Absolute excitation probabilities result in populations of $\nu=1$ and 2 close to those expected for complete vibrational thermalization with the surface (strong coupling case) despite the fact that the excitation happens on a sub-ps time-scale. We present a new kinetic model capable of describing the strong (as well as the weak) coupling case and, through detailed comparison with experiment, we show that electron mediated vibrational overtone excitation proceeds with similar intrinsic efficiency to single quantum excitation, limited only by the population of suitably energetic EHPs.

\section{The model $\dagger$}

The temperature dependence of vibrational excitation in molecular collisions at metal surfaces has long been known to follow an Arrhenius-like law, eqn (1), when the vibrational excitation mechanism involves energy transfer from EHPs. ${ }^{16}$

$$
P\left(\Delta E_{\mathrm{vib}}\right)=A e^{-\frac{\Delta E_{\mathrm{vib}}}{k_{\mathrm{B}} T_{\mathrm{S}}}}
$$

Here, $P$ is the excitation probability, $\Delta E_{\mathrm{vib}}$ is the vibrational excitation energy, $T_{\mathrm{S}}$ is the surface temperature and $k_{\mathrm{B}}$ is the Boltzmann constant. The pre-factor, $A$, describes the intrinsic coupling between EHPs and molecular vibration while the exponential term expresses, albeit in an approximate fashion, the statistical likelihood to find a thermally excited EHP of the correct energy to excite molecular vibration. Experimentally determining state-to-state pre-factors is one possible way to obtain the relative importance of vibrational overtone excitation, as the pre-factors can be considered the high- $T_{\mathrm{S}}$ limit, where the statistics of the EHP energy distribution are factored out. But this approach is potentially problematic.

For example, eqn (1) breaks down in the strong coupling case i.e. where $A$ is large or where $T_{\mathrm{S}}$ is high - as it cannot describe thermal equilibrium with the surface, ${ }^{31}$ which is clearly nonArrhenius.

$$
P_{\text {therm }}\left(\Delta E_{\text {vib }}\right)=e^{-\frac{\Delta E_{\text {vib }}}{k_{\mathrm{B}} T_{\mathrm{S}}}}\left(1-e^{-\frac{h \nu}{k_{\mathrm{B}} T_{\mathrm{S}}}}\right)
$$

Another limitation of the Arrhenius analysis represented by eqn (1) is the difficulty in distinguishing between direct vibrational overtone excitation, which is expected to have the Arrhenius form:

$$
P_{\text {direct }}(0-2)=A_{0,2} e^{-\frac{2 h \nu}{k_{\mathrm{B}} T_{\mathrm{S}}}}
$$

and sequential single quantum excitation which would have the form:

$$
\begin{aligned}
P_{\text {sequential }}(0-2) & =P_{\text {direct }}(0-1) * P_{\text {direct }}(1-2) \\
& =A_{0,1} e^{-\frac{h \nu}{k_{\mathrm{B}} T_{\mathrm{S}}}} A_{1,2} e^{-\frac{h \nu}{k_{\mathrm{B}} T_{\mathrm{S}}}}=A_{0,1} A_{1,2} e^{-\frac{2 h \nu}{k_{\mathrm{B}} T_{\mathrm{S}}}}
\end{aligned}
$$

Note that eqn (3) and eqn (4) exhibit the same temperature dependence and differ only in their pre-exponential factors.

To address these issues, we have developed a kinetic model for vibrational state changing events occurring in collisions with a metal surface that incorporates microscopic reversibility and a statistical treatment of the thermal energy distribution of metallic EHPs modelled as a Fermi gas of electrons in a three dimensional box. The model includes rate processes that describe one and two quantum excitation and de-excitation between the three lowest vibrational states: $\nu=0,1$ and 2 . In addition to describing deviations from eqn (1) associated with the transition to equilibrium with the surface in the strong coupling case, this model also predicts conditions where molecule-surface collisions result in multi-quantum excitation. Furthermore, the model suggests under what condition the efficiency of multi-quantum excitation will result from direct vibrational overtone up-pumping as opposed to sequential single quantum up-pumping events.

To test these ideas, we have carried out temperature dependent measurements from $T_{\mathrm{S}}=300-985 \mathrm{~K}$ of EHP mediated vibrational excitation probabilities for $\mathrm{NO}(\nu=1$ and 2) in collisions of $\mathrm{NO}(\nu=0)$ with a $\mathrm{Au}(111)$ surface. We show that application of eqn (1) to these data results in an ambiguous physical interpretation concerning the importance of overtone vibrational excitation. In contrast, not only are the data well described by the new model but clear physical insights emerge. Specifically, the absolute temperature dependent excitation probabilities of $\mathrm{NO}(\nu=0)$ to $\mathrm{NO}(\nu=1$ and 2$)$ are correctly captured by the model when the intrinsic coupling is roughly equal for both fundamental and overtone transitions. Furthermore, if the model is adjusted to eliminate direct overtone transitions, the data cannot be accurately reproduced.

We use a simple rate equation method to describe EHP mediated energy transfer. The model includes six rate processes:

$$
\begin{aligned}
& n_{0} \rightleftharpoons n_{1} \\
& n_{0} \rightleftharpoons n_{2} \\
& n_{1} \rightleftharpoons n_{2}
\end{aligned}
$$

with six rate constants: three for vibrational excitation:

$$
\begin{aligned}
& k_{0,1}=\xi_{0,1} * f_{1}\left(T_{\mathrm{S}}\right) \\
& k_{1,2}=\xi_{1,2} * f_{1}\left(T_{\mathrm{S}}\right) \\
& k_{0,2}=\xi_{0,2} * f_{2}\left(T_{\mathrm{S}}\right)
\end{aligned}
$$

and three for vibrational de-excitation:

$$
\begin{aligned}
& k_{1,0}=\xi_{1,0} * f_{-1}\left(T_{\mathrm{S}}\right) \\
& k_{2,1}=\xi_{2,1} * f_{-1}\left(T_{\mathrm{S}}\right) \\
& k_{2,0}=\xi_{2,0} * f_{-2}\left(T_{\mathrm{S}}\right)
\end{aligned}
$$


Here, $n_{i}$ is the instantaneous population of the $i^{\text {th }}$ vibrational state and $k_{i, j}$ is the rate constant for transitions from state $i$ to state $j$. Each rate process is modelled as a product of an intrinsic coupling strength, $\xi_{i, j}$ and an electronic statistical factor, $f_{\Delta v}\left(T_{\mathrm{S}}\right)$, which we will presently define. We assert on the basis of microscopic reversibility that $\xi_{i, j}=\xi_{j, i}$.

The electronic statistics are calculated as follows. We first assume that the Fermi function:

$$
F\left(\varepsilon ; T_{\mathrm{S}}\right)=\frac{1}{1+e^{\frac{\varepsilon-\varepsilon_{\mathrm{F}}}{k_{\mathrm{B}} T_{\mathrm{S}}}}}
$$

when combined with the density of states function of a spin- $1 / 2$ particle in a three dimensional box:

$$
\rho(\varepsilon)=2 \times 4 \pi \sqrt{\frac{2 m_{\mathrm{e}}^{3}}{h^{6}}} V \sqrt{\varepsilon}
$$

adequately describes the energy distribution of the electrons in the metal near the Fermi level. Here, $\epsilon_{\mathrm{F}}$ is the Fermi energy; $m_{\mathrm{e}}$ is the electron mass; $h$ is Planck's constant and $V$ is the volume of the metal. The electron distribution is then given by:

$$
p_{e}\left(\varepsilon ; T_{\mathrm{S}}\right)=F\left(\varepsilon, T_{\mathrm{S}}\right) * \rho(\varepsilon)
$$

while the hole distribution is:

$$
p_{\mathrm{h}}\left(\varepsilon ; T_{\mathrm{S}}\right)=\rho(\varepsilon)^{*}\left(1-F\left(\varepsilon, T_{\mathrm{S}}\right)\right)
$$

Using these distributions we define:

$$
f_{\Delta v}\left(T_{\mathrm{S}}\right)=\int_{\varepsilon=0}^{\infty} \mathrm{d} \varepsilon \int_{\varepsilon^{\prime}=0}^{\infty} \mathrm{d} \varepsilon^{\prime} p_{\mathrm{e}}\left(\varepsilon ; T_{\mathrm{S}}\right)^{*} p_{\mathrm{h}}\left(\varepsilon^{\prime} ; T_{\mathrm{S}}\right)^{*} \delta\left(\varepsilon-\varepsilon^{\prime}-\Delta v^{*} h \nu\right)
$$

where $\Delta \nu$ are the integers \pm 1 or \pm 2 .

The function $f_{\Delta \nu}\left(T_{\mathrm{S}}\right)$ represents the sum over all states where a thermally excited EHP is present whose relaxation energy is equal to the vibrational excitation, $\Delta \nu>0$. Similarly, it represents the sum over all electronic states where an electron and a higher energy hole is present capable of accepting the vibrational energy, $\Delta \nu<0 .{ }^{32}$

With these definitions of the rate constants, we solve the system of differential equations analytically using the Laplace transform method, ${ }^{33,34}$ assuming initial conditions where population is found only in $\nu=0$.

$$
\begin{aligned}
& \frac{\mathrm{d} n_{0}(t)}{\mathrm{d} t}=-\left(k_{0,1}+k_{0,2}\right) n_{0}(t)+k_{1,0} n_{1}(t)+k_{2,0} n_{2}(t) \\
& \frac{\mathrm{d} n_{1}(t)}{\mathrm{d} t}=k_{0,1} n_{0}(t)-\left(k_{1,0}+k_{1,2}\right) n_{1}(t)+k_{2,1} n_{2}(t) \\
& \frac{\mathrm{d} n_{2}(t)}{\mathrm{d} t}=k_{0,2} n_{0}(t)+k_{1,2} n_{1}(t)-\left(k_{2,0}+k_{2,1}\right) n_{2}(t)
\end{aligned}
$$

where:

$$
n_{0}(0)=1 \text { and } n_{1}(0)=n_{2}(0)=0
$$

Fig. 1 shows an example of solutions for the populations, $n_{1}(t)$ and $n_{2}(t)$, derived from the rate model. Here we have assumed


Fig. 1 The approach to thermal equilibrium at $T_{\mathrm{S}}=1000 \mathrm{~K}$. Upper panel: The solution to the kinetic equations for $\xi_{0,2}=0.69^{*} \xi_{1,2}$ and $\xi_{1,2}=\xi_{0,1}=1$ for the population, $n$, of $\nu=1$ (dashed) and $\nu=2$ (solid). The dot-dashed lines show the thermal populations for the two vibrational states. The vertical line at $\tau_{\text {eff }}=0.96$ represents the effective collision time derived from comparison to the $\mathrm{NO} / \mathrm{Au}$ scattering data. See text. Although not shown, the population of $\nu=0$ also approaches the thermal value on this time-scale. Lower panel: for the case of $\xi_{0,2}=0.69 * \xi_{1,2}$ and $\xi_{1,2}=\xi_{0,1}=0.01$.

certain reasonable values (see figure caption) for the intrinsic coupling strengths, $\xi_{i, j}$, in order to examine the short and long time behaviour of the model. While the precise solution is sensitive to the choice of coupling strengths, the qualitative fact that the populations for $\nu=1$ and 2 are initially zero and grow to a steady-state thermal population distribution - shown as dotdashed lines - is not.

It is useful to envision a physical picture to accompany the mathematical solutions obtained from the model; perhaps most importantly as this helps illuminate the assumptions of the model. We envision a molecule colliding with a surface in a direct scattering process. The model assumes that non-adiabatic coupling occurs with a constant magnitude. Thus as the molecule approaches the surface, there is some distance where the coupling turns on like a step function. It is then further assumed that the coupling remains constant during an "effective" collision time, $\tau_{\text {eff. }}$ We will show below that the vertical line in the upper panel of Fig. 1 represents the $\mathrm{NO} / \mathrm{Au}$ scattering in this work and corresponds to an effective collision time of about $\tau_{\text {eff }}=400 \mathrm{fs}$, within which the system comes approximately halfway to the equilibrium limit. We dub this scattering regime the strong coupling case. 
Fig. 1 also shows the behaviour of the model when the coupling strength is reduced by 100 fold (lower panel), conditions that could be achieved for example by reducing the incidence energy of translation. Here, one obtains analogous growth curves that terminate in a steady state thermal population; however, the time-scale required to reach the steady state is 100 times longer. For a similar value of $\tau_{\text {eff }}$, only about one-100th of the vibrational excitation would be observed, a regime we describe as the weak coupling case.

One may note from this comparison that when we arbitrarily fix $\xi_{0,1}$, we may vary the population transfer by adjusting $\tau_{\text {eff }}{ }^{35}$ Thus in all of the comparisons with real data that we will make below, $\xi_{0,1}=1$, as in Fig. 1 (upper panel). We then adjust the effective collision time, $\tau_{\text {eff }}$, to describe the overall strength of coupling. We can then vary the magnitudes of $\xi_{1,2}$ and $\xi_{0,2}$ with respect to $\xi_{0,1}$ to explore the relative importance of single and multi-quantum processes.

\section{Observation of multi-quantum vibrational excitation}

The experimental apparatus has been previously described in detail. ${ }^{36}$ In brief, we produce supersonic molecular beam pulses ${ }^{37}$ of NO with a translational energy of incidence, $E_{\mathrm{I}} \sim 0.9 \mathrm{eV}$,

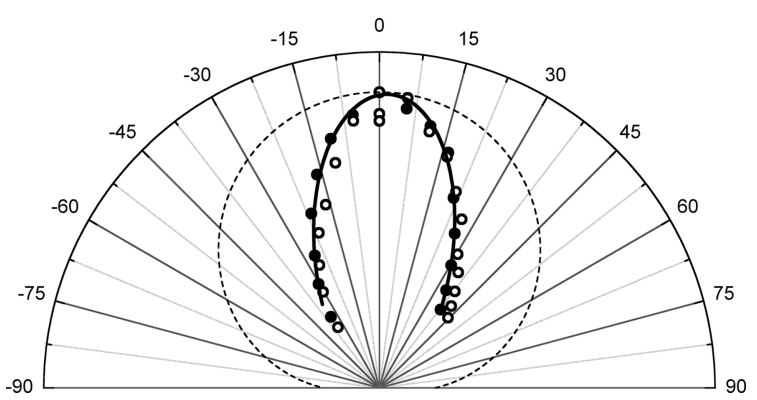

Fig. 2 Angular distribution of the scattered beams, $\nu=2$ (solid circles), $\nu=1$ (open circles). The solid line is a $\cos ^{7.3}\left(\theta-1.6^{\circ}\right)$ function and the dashed line is a $\cos \theta$ function, expected if trapping/desorption was the predominant process. The absolute intensities of the two angular distributions are normalized with respect to each other to emphasize the shape of the scattered angular distribution.

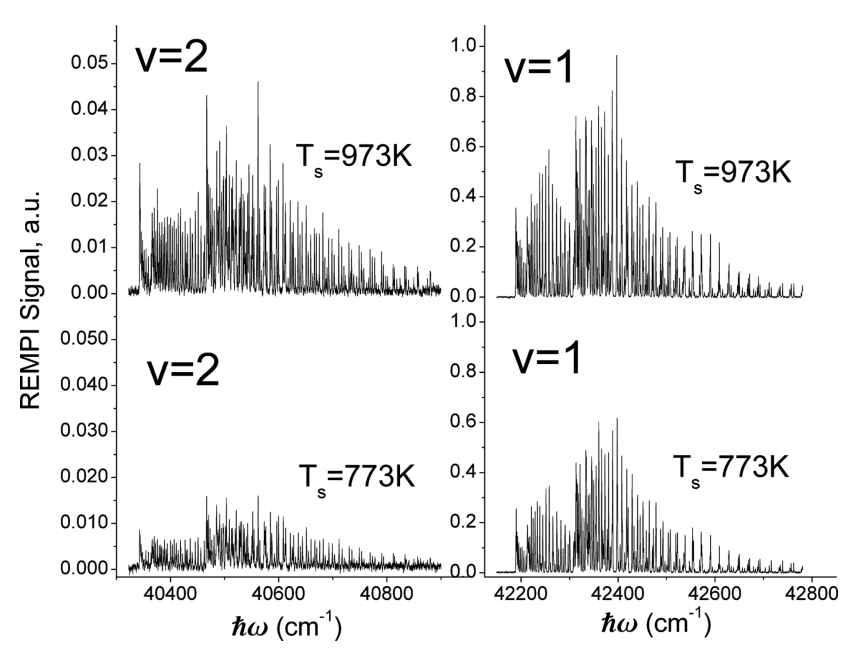

Fig. 3 Examples of REMPI spectra ${ }^{47}$ of surface-scattered NO. which collide at normal incidence with a $\mathrm{Au}(111)$ surface, whose temperature can be varied from 100-985 K within minutes ${ }^{38}$ and is held in an ultra-high vacuum chamber. ${ }^{39}$ State-specific REMPI $^{40}$ detection of NO is accomplished with UV laser pulses ${ }^{41}$ and an ion detection system. ${ }^{42}$ By varying the wavelength, laser/molecular-beam pulse timing and by translating the laser beam parallel to the surface, we can measure: rotationally resolved REMPI spectra, ${ }^{43}$ temporal profiles $^{44}$ and scattering angular distributions, respectively. ${ }^{45}$

Fig. 2 shows angular distributions for scattered NO molecules that have undergone vibrational excitation in collisions with $\mathrm{Au}(111)$. The angular distributions peak at the specular angle ( $1.6^{\circ}$ from the surface normal) and exhibit a FWHM of less than $40^{\circ}$. They are much narrower than a $\cos \theta$ distribution characteristic of trapping/desorption and indicate a direct, singlebounce vibrational excitation mechanism.

Fig. 3 shows rotationally resolved REMPI spectra of scattered $\mathrm{NO}(\nu=1 \text { and } 2)^{25}$ at two surface temperatures. The scattered molecules are rotationally hot $\left(T_{\text {rot }} \sim 700 \mathrm{~K}\right)$ compared to the incident beam $\left(T_{\text {rot }} \sim 10 \mathrm{~K}\right)^{46}$ and $T_{\text {rot }}$ is found to be only weakly dependent on $T_{\mathrm{S}}$, consistent with a direct single-bounce scattering mechanism. A glance at the figure reveals that the increase in REMPI signal with surface temperature is strong for both $\nu=1$ and 2 but stronger for $\nu=2$.

To derive the absolute vibrational excitation probabilities, data like those shown in Fig. 3 probing $\nu=0,1^{48}$ and 2 were recorded between $T_{\mathrm{S}}=300$ and $985 \mathrm{~K}$, integrating the entire spectral intensity in each vibrational band. These integrated intensities were corrected for differences in temporal and angular distributions and normalized to differences in the laser power and MCP voltage. ${ }^{49} \mathrm{We}$ also corrected for differences between each band's Franck-Condon factor. ${ }^{50}$ Ratios of these corrected signal intensities $\left(S_{\nu}\right)$ yield absolute excitation probabilities, $P_{\nu}{ }^{51}$

$$
P_{\nu}=\frac{S_{v}}{S_{0}}
$$

The derived values of $P_{\nu}$ are shown in Fig. 4. At all surface temperatures in this work, $P_{1}$ is at least $10 \times$ larger than $P_{2}$.



Fig. 4 Vibrational excitation probabilities $v$ s. surface temperature. $\nu=2$ (closed circles), $\nu=1$ (open circles). The thick lines are the canonical Arrhenius fits and the thin lines are the floating Arrhenius fits, constants for which are presented in Table 1. See text. The dot-dashed lines are the thermal limit. The data have been culled to eliminate statistical outliers. ${ }^{52}$ 
Table 1 Arrhenius fitting of vibrational excitation data

\begin{tabular}{|c|c|c|c|c|}
\hline & $\begin{array}{l}\text { Fitting } \\
\text { parameters }\end{array}$ & $\begin{array}{l}\text { Canonical } \\
\text { Arrhenius fit }\end{array}$ & $\begin{array}{l}\text { Floating } \\
\text { Arrhenius fit }\end{array}$ & $\begin{array}{l}\text { Kinetic model } \\
\text { Arrhenius fit }^{a}\end{array}$ \\
\hline$\nu=1$ & $\begin{array}{l}A \\
E_{\mathrm{a}} / \mathrm{cm}^{-1} \\
\mathrm{RMS}^{b}\end{array}$ & $\begin{array}{l}0.39 \\
1904 \\
0.0014\end{array}$ & $\begin{array}{l}0.34 \\
1814 \\
0.0014\end{array}$ & $\begin{array}{l}0.41 \\
1942 \\
-\end{array}$ \\
\hline$\nu=2$ & $\begin{array}{l}A \\
E_{\mathrm{a}} \\
\mathrm{RMS}^{b}\end{array}$ & $\begin{array}{l}0.46 \\
3808 \\
0.00028\end{array}$ & $\begin{array}{l}0.83 \\
4189 \\
0.00028\end{array}$ & $\begin{array}{l}0.45 \\
3798 \\
-\end{array}$ \\
\hline
\end{tabular}

${ }^{a}$ The coupling strength was $\tau_{\text {eff }}=0.96$ and the state-to-state coupling was $\xi_{0,2}=0.69^{*} \xi_{1,2}$ and $\xi_{1,2}=\xi_{0,1}=1 .{ }^{b}$ The RMS is defined as follows: $\sqrt{\frac{\sum_{j=1}^{N}\left[A e^{-\frac{E_{\mathrm{a}}}{k_{\mathrm{B}} T_{j}}}-n_{i}\left(T_{j}\right)\right]^{2}}{N-1}}$

At $T_{\mathrm{S}}=985 \mathrm{~K}, P_{1}=0.025$, which compares well with the result $P_{1}=0.07 \pm 0.05$ reported for $\mathrm{NO}$ scattering from $\mathrm{Ag}(111){ }^{16,53}$ Also shown in Fig. 4 are the thermal limits - eqn (2) - as well as Arrhenius functions - eqn (1) - obtained by fitting in two different ways. See below. The Arrhenius activation energies (Table 1) are close to $\Delta E_{\text {vib }}$ in all cases; however, as discussed above in relation to eqn (3), this is not sufficient to establish the importance of direct vibrational overtone excitation.

\section{Comparing observation to theory: evidence of vibrational overtone excitation}

We systematically studied the behaviour of the model in close comparison to the experimental data. Although there appear to be many adjustable parameters, i.e. $\tau_{\mathrm{eff}}, \xi_{0,1}, \xi_{1,2}$ and $\xi_{0,2}$, we easily find a robust fit to the data. As described above, the population transfer is determined only by the product of $\tau_{\text {eff }}$ with $\xi_{0,1}$. Furthermore, we find that the fit to the data is only weakly dependent on $\xi_{1,2}$. Thus in practice, for a fixed value of $\xi_{0,1}$, only $\tau_{\text {eff }}$ and $\xi_{0,2}$ affect the quality of the fit to the data. Furthermore, these two fitting parameters are nearly independent of one another, $\tau_{\text {eff }}$ effectively controlling the probability of $0-1$ vibrational excitation and $\xi_{0,2}$ the branching fraction to $\nu=2$.

Fig. 5 shows the best $\mathrm{fit}^{54}$ to the data. Comparing to the thermal limit (dot-dashed curves) and noting the derived value of $\tau_{\text {eff }}=0.96$, one can clearly see that NO scattering from $\mathrm{Au}(111)$ at this incidence energy is an example of strong electronically non-adiabatic coupling. Referring to Fig. 1, one can see that this value of $\tau_{\text {eff }}$ represents a coupling strength that brings the system roughly halfway to the thermal limit.

In passing, we note that one may estimate $\tau_{\text {eff }}$ and hence the absolute time-scale of the plot in the upper panel of Fig. 1. A recent paper of ours identified a critical distance for vibrationally promoted electron emission, ${ }^{21} Z_{\mathrm{C}} \sim 5 \AA$, which despite several important differences we take to be characteristic of the interaction distance for vibrational excitation in this work at least for the purpose of estimation. From the measured velocity of the NO molecular beam, $v_{\mathrm{NO}}$, we estimate the round trip time of the collision, $\tau_{\text {eff }}$, within a constant velocity approximation:



Fig. 5 Comparison of experimental NO vibrational excitation probabilities $\left(P_{v}\right)$ to the kinetic model. The effective collision time was $\tau_{\text {eff }}=0.96$, and the state-to-state coupling was $\xi_{0,2}=0.69 * \xi_{1,2}$ and $\xi_{1,2}=\xi_{0,1}=1$. The closed and open circles are experimental data for $\nu=2$ and 1, respectively. The solid and dashed lines are the results of the kinetic model. The dot-dashed lines are the thermal expectation.

$$
\tau_{\text {eff }}=\frac{2 * Z_{\mathrm{C}}}{V_{\mathrm{NO}}} \approx 400 \mathrm{fs}
$$

This leads to an estimate of the time units in Fig. 1 (upper panel); namely, $420 \mathrm{fs}$. This analysis implies that electron mediated energy transfer provides a path to molecule-surface equilibrium on the sub-ps time-scale, ${ }^{55}$ consistent with vibrational lifetime measurements for small molecules on metal surfaces. ${ }^{17}$ This is to be compared with vibrational relaxation to phonons, which is several orders of magnitude slower. ${ }^{22-24}$

In characterizing the excitation to $\mathrm{NO}(\nu=2)$ it is important to distinguish between direct and sequential excitation. Since production of $\mathrm{NO}(\nu=1)$ is always at least $10 \times$ more efficient than production of $\operatorname{NO}(\nu=2)$ under our conditions, it is reasonable to think that $\mathrm{NO}(\nu=2)$ might be produced by two single-quantum excitation steps and not by a vibrational overtone mechanism.

Fig. 6 shows the impact on the fit if vibrational overtone pumping is neglected, that is setting $\xi_{0,2}=0$. While this change

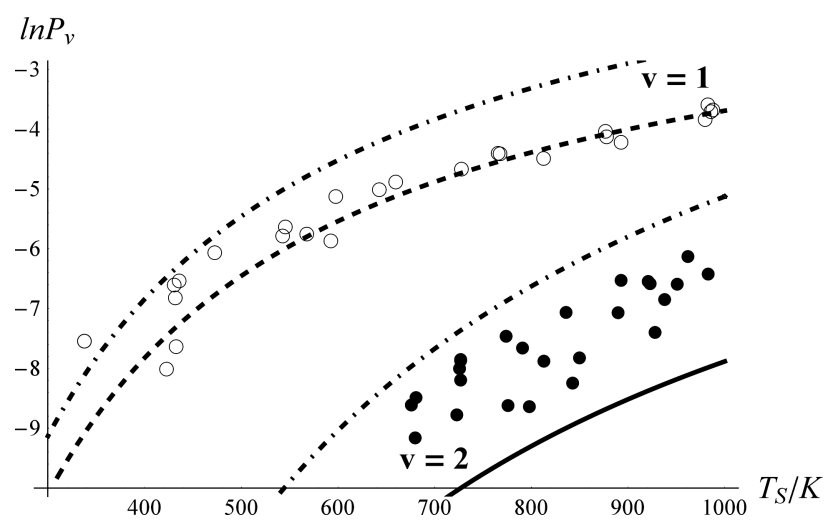

Fig. 6 The importance of vibrational overtone excitation. This is the resulting fit if $\xi_{0,2}=0$. Otherwise, the fitting parameters are identical to those of Fig. 5. The Arrhenius parameters obtained for the 0-2 excitation in the sequential mechanism (solid line) are $A=0.11$ and $E_{\mathrm{a}}=3931 \mathrm{~cm}^{-1}$. 
has no noticeable effect on the predicted $\nu=1$ populations, the $\nu=2$ excitation probability is dramatically underestimated. To obtain a reasonable fit when overtone pumping is neglected $\xi_{1,2}$ has to become unreasonably large, $\xi_{1,2}>20 * \xi_{0,1}$. There is no reason to think that $\xi_{1,2}$ should be much larger than $\xi_{0,1}$, as indicated by the full fit. In contrast, one may start with the best fit parameters of Fig. 5 and change $\xi_{1,2}$ to 0 , with no detectable effect on the fit.

Therefore, we conclude that $\nu=2$ is predominantly populated by direct vibrational overtone excitation. Indeed, the best fit results $-\xi_{0,2}=0.69 * \xi_{0,1}$ - suggest that the multi-quantum vibrational excitation is about as strong as single quantum excitation. This contrasts starkly with optical overtone transition probabilities. For example for NO, the 1st optical overtone strength is 0.015 that of the fundamental. ${ }^{56}$

Prior to the kinetic model presented in this edge article, it has been customary to compare the $T_{\mathrm{S}}$-dependence of vibrational excitation data to an Arrhenius relation:

$$
P_{\nu}=A e^{-\frac{E_{a}}{k_{\mathrm{B}} T_{\mathrm{S}}}}
$$

Evidence for EHP mediated vibrational energy transfer has been inferred when, by fitting data like that shown in Fig. 4, $E_{\mathrm{a}} \sim \Delta E_{\mathrm{vib}}$ is obtained. One may carry out such fitting optimizing both $A$ and $E_{\mathrm{a}}$ as was done by Watts et al. ${ }^{29}$ We refer to this as the "floating Arrhenius fit" method. The floating fit makes no assumptions about the outcome of the model and may, in general, fit the data better over wide temperature ranges in the strong coupling case. Alternatively, one may assume $E_{\mathrm{a}}=\Delta E_{\mathrm{vib}}$ and optimize the fit by varying $A$ only as was done by Rettner et al. ${ }^{16} \mathrm{We}$ call this the "canonical Arrhenius fit" method.

Table 1 shows derived results for $A$ and $E_{\mathrm{a}}$ using both the canonical and floating methods as well as the root-mean-square (RMS) deviations of the fits. Also see Fig. 4. It is immediately obvious that one cannot distinguish the canonical from the floating fit based on a goodness of fit criterion. This ambiguity results from the finite noise associated with the data and the resulting statistical correlation between $A$ and $E_{\mathrm{a}}$.

This ambiguity limits our ability to interpret the meaning of the derived parameters, $A$ and $E_{\mathrm{a}}$. According to the canonical fit results, the ratio of the $A$-factors is $0.46 / 0.39=1.2$, while the floating fit gives a larger ratio of $\sim 2.5$. Interestingly, by fitting the results of the kinetic model to an Arrhenius form, letting both $A$ and $E_{\text {a }}$ be optimized, we find excellent agreement with the canonical results. See the last column of Table 1 . This reflects the underlying correctness of the canonical Arrhenius picture, which can, in fact, be written in terms of the kinetic model of this edge article, a topic that will be treated in detail in a future publication. ${ }^{57}$

\section{Conclusions}

We have shown that electron mediated vibrational overtone excitation can be observed experimentally and, employing a new kinetic model in comparison to the data, that the intrinsic overtone transition strength is of the same order as that of the fundamental transition. While this study looked specifically at thermal EHP populations, these results imply that many quanta of vibration might be excited in a molecule due to a surface collision, if the electron energy distribution in the solid were suitably prepared, for example by photo-excitation. This might provide a subtle approach to circumventing optical selection rules for vibrational excitation. This work furthermore suggests that the electron hopping mechanism used to explain multiquantum vibrational relaxation of $\mathrm{NO}(\nu=15)$ in collisions with a $\mathrm{Au}(111)$ surface $^{\mathbf{1 0}}$ can also be applied to describe vibrational excitation of $\mathrm{NO}(\nu=0)$.

\section{Acknowledgements}

The authors gratefully acknowledge financial support from the Department of Energy Office of Basic Energy Sciences, under Grant No. DE-FG02-03ER15441 as well as the Partnership for International Research and Education-for Electronic Chemistry and Catalysis at Interfaces-NSF Grant No. OISE0530268. AMW would like to acknowledge the Max Planck Institute for Biophysical Chemistry and Institute for Physical Chemistry at the University of Göttingen for support during a sabbatical stay that accelerated the appearance of this edge article.

\section{Notes and references}

1 G. A. Somorjai, H. Frei and J. Y. Park, J. Am. Chem. Soc., 2009, 131, 16589-16605.

2 B. A. Gregg, J. Phys. Chem. B, 2003, 107, 4688-4698.

3 A. Ennaoui, S. Fiechter, C. Pettenkofer, N. Alonsovante, K. Buker, M. Bronold, C. Hopfner and H. Tributsch, Sol. Energy Mater. Sol. Cells, 1993, 29, 289-370.

4 J. Rempel, J. Greeley, L. B. Hansen, O. H. Nielsen, J. K. Norskov and M. Mavrikakis, J. Phys. Chem. C, 2009, 113, 20623-20631.

5 K. Reuter and M. Scheffler, Phys. Rev. B, 2002, 65, 035406.

6 B. Gergen, H. Nienhaus, W. H. Weinberg and E. W. McFarland, Science, 2001, 294, 2521-2523.

7 J. Y. Park and G. A. Somorjai, ChemPhysChem, 2006, 7, 1409-1413.

8 R. D. Beck, P. Maroni, D. C. Papageorgopoulos, T. T. Dang, M. P. Schmid and T. R. Rizzo, Science, 2003, 302, 98-100.

9 E. H. G. Backus, A. Eichler, A. W. Kleyn and M. Bonn, Science, 2005, 310, 1790-1793.

10 N. Shenvi, S. Roy and J. C. Tully, Science, 2009, 326, 829-832.

11 P. Nieto, E. Pijper, D. Barredo, G. Laurent, R. A. Olsen, E. J. Baerends, G. J. Kroes and D. Farias, Science, 2006, 312, 86-89.

12 G. J. Kroes, Prog. Surf. Sci., 1999, 60, 1-85.

13 L. B. F. Juurlink, D. R. Killelea and A. L. Utz, Prog. Surf. Sci., 2009, 84, 69-134.

14 A. M. Wodtke, D. Matsiev and D. J. Auerbach, Prog. Surf. Sci., 2008, 83, 167-214.

15 A. M. Wodtke, J. C. Tully and D. J. Auerbach, Int. Rev. Phys. Chem., 2004, 23, 513-539.

16 C. T. Rettner, F. Fabre, J. Kimman and D. J. Auerbach, Phys. Rev. Lett., 1985, 55, 1904-1907.

17 V. Krishna and J. C. Tully, J. Chem. Phys., 2006, 125, 6.

18 Y. H. Huang, C. T. Rettner, D. J. Auerbach and A. M. Wodtke, Science, 2000, 290, 111-114.

19 J. D. White, J. Chen, D. Matsiev, D. J. Auerbach and A. M. Wodtke, Nature, 2005, 433, 503-505.

20 J. D. White, J. Chen, D. Matsiev, D. J. Auerbach and A. M. Wodtke, J. Chem. Phys., 2006, 124, 13.

21 N. H. Nahler, J. D. White, J. Larue, D. J. Auerbach and A. M. Wodtke, Science, 2008, 321, 1191-1194.

22 H. C. Chang and G. E. Ewing, Phys. Rev. Lett., 1990, 65, 2125-2128.

23 M. Korolik, M. M. Suchan, M. J. Johnson, D. W. Arnold, H. Reisler and C. Wittig, Chem. Phys. Lett., 2000, 326, 11-21.

24 A. M. Wodtke, Y. H. Huang and D. J. Auerbach, J. Chem. Phys., 2003, 118, 8033-8041.

25 A. M. Wodtke, Y. H. Huang and D. J. Auerbach, Chem. Phys. Lett., 2005, 414, 138-142. 
26 A. M. Wodtke, H. Yuhui and D. J. Auerbach, Chem. Phys. Lett., 2005, 413, 326-330.

27 M. Drabbels and A. M. Wodtke, J. Chem. Phys., 1997, 106, 3024 3028.

28 J. W. Gadzuk and S. Holloway, Phys. Rev. B, 1986, 33, 4298-4300.

29 E. K. Watts, J. L. W. Siders and G. O. Sitz, Surf. Sci., 1997, 374, 191 196.

30 It should be noted that multi-quantum vibrational excitation has been reported for collisions of $\mathrm{NH}_{3}$ with $\mathrm{Au}(111)$, but there the mechanism is thought to be purely mechanical in nature. See: B. D. Kay, T. D. Raymond and M. E. Coltrin, Phys. Rev. Lett., 1987, 59, 2792-2794.

31 Eqn (2) is derived from the canonical partition function of the simple harmonic oscillator with vibrational frequency $\nu$, where $h$ is Planck's constant.

32 To adapt the model to an anharmonic oscillator would require slight changes where, for example, the statistical factor for 2-1 transitions would be slightly different than for 1-0 transitions.

33 B. Davis, Integral Transforms and their Applications, 3rd edn, Springer Verlag, New York, 2002.

34 G. A. Korn and T. M. Korn, Mathematical Handbook for Scientists and Engineers, 2nd edn, McGraw-Hill Companies, New York, 1967.

35 This turns out to be a practical way to implement the model, but means that the time units are no longer absolute. One may establish absolute time units in a post-facto analysis - vide infra.

36 Q. Ran, D. Matsiev, A. M. Wodtke and D. J. Auerbach, Rev. Sci. Instrum., 2007, 78, 104104.

$372.5 \%$ NO seeded in $\mathrm{H}_{2}$ was expanded through a piezoelectrically actuated pulsed valve with a stagnation pressure near $3 \mathrm{~atm}$. The valve opening time was $\sim 60 \mu \mathrm{s}$. The molecular beam is skimmed and passes through two regions of differential pumping before entering an ultra high vacuum surface science chamber.

38 The gold crystal can be resistively heated by passing electric current through its tungsten support wires and conductively cooled with liquid $\mathrm{N}_{2}$.

39 The base pressure in the surface chamber is $\sim 1 \times 10^{-10}$ Torr and reaches $\sim 1 \times 10^{-9}$ Torr with the molecular beam running. The main surface impurity is carbon. The surface is cleaned by sputtering with $3 \mathrm{kV} \mathrm{Ar}{ }^{+}$for at least $30 \mathrm{~min}$ and subsequently annealed at $1000 \mathrm{~K}$ for at least $30 \mathrm{~min}$. The surface cleanliness was verified using Auger electron spectroscopy. Based on the Auger spectra we estimate the impurities to be below $3 \%$ of a monolayer of coverage after sputtering and annealing. Furthermore, we measured before and after scattering and never found evidence for C-building up over the several hours measurement time.

$401+1$ resonance enhanced multiphoton ionization (REMPI) of $\mathrm{NO}(\nu=1)$ was accomplished via $\mathrm{A}-\mathrm{X}(0,1)$ transition using coumarin 480. $\mathrm{NO}(\nu=2)$ REMPI spectra were recorded via A-X $(0,2)$ transition using coumarin 503 , sometimes in a mixture with coumarin 480. $\mathrm{NO}(\nu=0)$ REMPI spectra, which were necessary for deducing the absolute excitation probabilities were monitored via $\mathrm{A}-\mathrm{X}(0,0)$ transition, using coumarin 450 . The dye laser is pumped with the third harmonic of a Nd:YAG laser. The output of the dye laser was doubled in a BBO crystal.

$415 \mathrm{~ns}$ duration, $2-4 \mathrm{~mJ}$ pulse energy, $0.5 \mathrm{~cm}^{-1}$ bandwidth and $\sim 250 \mathrm{~nm}$ wavelength.

42 The ions resulting from REMPI are guided to a two-stage microchannel plate (MCP) detector by a repeller and two electrostatic focusing lenses.
43 The rotational distributions of scattered $\mathrm{NO}(\nu=0,1,2)$ were recorded at the peaks of the temporal and angular distributions.

44 Temporal profiles were recorded by fixing the dye laser's wavelength on a specific rotational line and varying the delay between the laser and molecular beam pulses.

45 The temporal and angular distributions were independent of the chosen rotational transition.

46 Rotationally resolved $1+1$ REMPI spectra of NO were recorded via the $\mathrm{A}\left(\nu^{\prime}=0\right) \leftarrow \mathrm{X}\left(\nu^{\prime \prime}=0,1,2\right)$ bands after scattering from the surface. These bands were chosen because they are spectrally well isolated. Thus, we may integrate the measured REMPI intensity over the entire rotational distribution of a specific vibrational state. This simplifies the data analysis, eliminating the need to take into account variations of the rotational temperature in the scattered NO that results from changing the surface temperature and/or vibrational state.

47 The $x$-axis is the one-photon energy of the $1+1$ REMPI process.

48 For measurements of $\nu=1$, a small background was subtracted that arises from rotationally inelastic scattering of thermally populated $\mathrm{NO}(\nu=1)$ present in the molecular beam. The background level was determined by extrapolating the surface temperature dependence of the $\operatorname{NO}(\nu=1)$ signal to $0 \mathrm{~K}$ using a suitable Arrhenius expression.

49 Angular and temporal profiles of the scattered $\operatorname{NO}(\nu=0,1,2)$ were measured for the entire range of surface temperatures used in this work. The $1+1$ REMPI signal of the scattered $\operatorname{NO}(\nu=0,1,2)$ was found to vary linearly with the laser intensity $(2-4 \mathrm{~mJ})$, The voltage across the MCP was adjusted for each vibrational state with respect to the scattered signal intensity to avoid saturation. The variation of a small scattered signal with the voltage across the MCP detector was measured over the entire range of the MCP voltages used in this work to allow for signal normalization under different detection conditions.

50 J. Luque and D. R. Crosley, J. Chem. Phys., 1999, 111, 7405-7415.

51 The outgoing velocity of the scattered molecules was assumed to be independent of vibrational state. Thus no flux-density correction was performed. See: I. Rahinov, R. Cooper, C. Yuan, X. M. Yang, D. J. Auerbach and A. M. Wodtke, J. Chem. Phys., 2008, 129.

52 The term "culled" refers to a statistical analysis that was carried out on the data to determine if any points are statistical outliers. To accomplish this a canonical fit to the data was carried out and the standard deviation of the fit to the data was determined. Any points that were more that $2.5 \sigma$ from the canonical Arrhenius curve were then eliminated and the canonical fitting was repeated. This process was iterated until all points were within $2.5 \sigma$ of the canonical Arrhenius fit. This resulted in removal of 2 points for the $\nu=2$ data and 5 points for the $\nu=1$ data. If errors were random, points outside $2.5 \sigma$ represent $0.04 \%$ of the distribution.

53 Absolute excitation probabilities for $\mathrm{NO}$ on $\mathrm{Cu}(110)$ appear close to or beyond the thermal limit, possibly reflecting the difficulty of the measurements. See ref. 29.

54 The root mean square deviation of the fit was minimized by hand

55 The 1/e time to equilibrium can be found easily and is $\sim 700 \mathrm{fs}$.

56 Y. P. Lee, S. L. Cheah and J. F. Ogilvie, Infrared Phys. Technol., 2006, 47, 227-239.

57 The pre-factor, $A$, is related to the channel specific coupling of molecular vibration to EHPs. In a soon to be published paper, we will present simple analytical expressions for the Arrhenius constants in terms of the fundamental parameters of the kinetic model of this paper. 